\title{
Temporal and spectral control of the $X$-ray pulses in a resonant medium with a modulated transition frequency
}

Farit Vagizov, Vladimir Antonov, llias Khairulin, Yevgeny Radeonychev, Kyong-Chol Han, et al.

Farit Vagizov, Vladimir Antonov, Ilias Khairulin, Yevgeny Radeonychev, KyongChol Han, Olga Kocharovskaya, "Temporal and spectral control of the X-ray pulses in a resonant medium with a modulated transition frequency," Proc. SPIE 11886, International Conference on X-Ray Lasers 2020, 118860 (8 July 2021); doi: 10.1117/12.2593321 


\title{
Temporal and Spectral Control of the X-ray Pulses in a Resonant Medium with a Modulated Transition Frequency
}

\author{
Farit Vagizov a,b, Vladimir Antonov ${ }^{\mathrm{c}}$, Ilias Khairulin ${ }^{\mathrm{c}, \mathrm{d}}$, Yevgeny Radeonychev ${ }^{\mathrm{c}, \mathrm{d}}$, Kyong-Chol Han ${ }^{\mathrm{a}}$, \\ Olga Kocharovskaya ${ }^{* a}$ \\ ${ }^{a}$ Department of Physics and Astronomy, Texas A\&M University, 4242 TAMU, 578 University Dr., \\ College Station, TX 77843, USA; ${ }^{\mathrm{b}}$ Kazan Federal University, 18 Kremlyovskaya str., Kazan, Russia \\ 420008; 'Institute of Applied Physics of the Russian Academy of Sciences, 46 Ulyanov str., Nizhny \\ Novgorod, Russia 603950; ${ }^{\mathrm{N}}$ N. I. Lobachevsky State University of Nizhny Novgorod, 23 Gagarin \\ Avenue, Nizhny Novgorod, Russia 603950
}

\begin{abstract}
We discuss a method for controlling the spectral and temporal characteristics of $\mathrm{x}$-ray radiation produced by a radioactive or synchrotron Mössbauer source via its propagation through an optically thick sample of resonant nuclei with a modulated transition frequency. Such modulation is achieved via a Doppler frequency shift due to vibration of the recoilless absorber. We show that this technique can be used both for effective elimination of the resonant absorption (acoustically induced transparency) and temporal shaping of an individual photon, including the production of short pulses. A similar technique can be used for formation and amplification of attosecond pulses in the active medium of a plasma-based x-ray laser, where the resonant transition frequency of ions is modulated by a sufficiently strong infrared field.
\end{abstract}

Keywords: X-ray quantum optics, frequency modulation, Mössbauer effect, ultrashort pulse formation, induced transparency

\section{INTRODUCTION}

A new field of research on a border of quantum optics and x-ray optics, called quantum x-ray optics, has emerged recently $^{1,2}$. While quantum optics deals with the interaction of single optical photons with the ensembles of quantum emitters (atoms, molecules, rare-earth-ions doped crystals, color centers in diamond, quantum dots, etc.), quantum x-ray optics deals accordingly with the interaction of $\mathrm{x}$-ray photons with an ensemble of nuclei. The attractive features of $\mathrm{x}$-ray photons with respect to optical photons, based on their higher energy and accordingly lower wavelength, are the ability to detect them with much higher efficiency and focus them to a much tighter spot (principally limited by the wavelength).

Coherent control of the resonant interaction of the signal field with the quantum emitters is the basis of quantum optics. One of the widely used coherent control techniques in the optical range is based on driving an adjacent transition with a sufficiently strong field in the so-called lambda configuration. This results in suppression of the resonant absorption of the signal field via Autler-Townes splitting (ATS) or electromagnetically induced transparency (EIT) $)^{3,4}$.

The direct implementation of this technique for controlling the resonant interaction of $\mathrm{x}$-ray radiation $(1-100 \mathrm{keV})$ with nuclei is challenging, since typically no transitions which could be efficiently driven by existing coherent sources (such as lasers or high-harmonic generation (HHG) sources) are available in nuclei (although ATS at $\sim 10 \mathrm{keV}$ nuclear transitions was observed with the microwave and DC driving ${ }^{5,6}$ and some analog of EIT in nuclear sandwich structures inside $\mathrm{x}$-ray waveguides was also realized $^{7}$ ).

*kochar@physics.tamu.edu; phone (979) 845-7717; fax (979) 845-2590; physics.tamu.edu

International Conference on X-Ray Lasers 2020, edited by Davide Bleiner,

Proc. of SPIE Vol. 11886, 118860I · @ 2021 SPIE · CCC code:

0277-786X/21/\$21 - doi: $10.1117 / 12.2593321$

Proc. of SPIE Vol. 11886 118860I-1 
In this work we briefly review a different technique for coherent control of x-rays-matter interaction, based on modulation of the frequency of the resonant transition. In the case of the nuclear transitions, the frequency modulation can be produced via the Doppler effect $\left(\omega=\omega_{21}+(2 \pi R \Omega / \lambda) \cos (\Omega t+\phi)\right.$, where $\omega$ and $\omega_{21}$ are the frequencies of modulated and unperturbed nuclear transition, and $\lambda$ is the radiation wavelength) due to vibration of the resonant absorber: $\Delta z=R \sin (\Omega t+\phi)$ (where $\Omega, R$, and $\phi$ are the frequency, amplitude, and initial phase of the absorber vibration, accordingly), induced by the piezoelectric transducer attached to the absorber and driven by harmonically varying voltage (see Fig. 1).

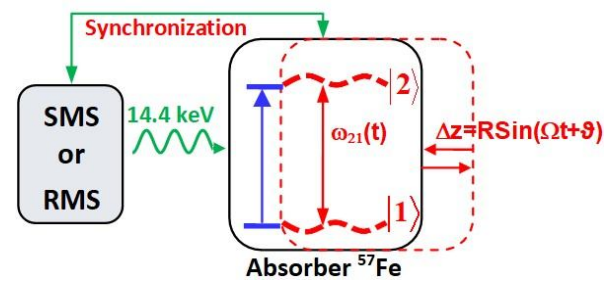

Figure 1. (Color online) Scheme of the emission (left side) from the radioactive Mössbauer source (RMS) or synchrotron Mössbauer source (SMS) and recoilless resonant transmission (right side) of 14.4-keV photons through the vibrating ${ }^{57} \mathrm{Fe}$ absorber. The photon resonant absorption in motionless absorber (blue lines) can be strongly altered if the absorber harmonically vibrates as a whole (piston-like vibration) along the photon propagation direction (marked in red) due to periodic temporal modulation of $|1\rangle \rightarrow|2\rangle$ transition frequency, $\omega_{21}(t)$ (dashed red curves), because of the Doppler effect. The emission of photons can be synchronized with the initial phase of the absorber vibration.

\section{COHERENT CONTROL OF THE TRANSMITTED RADIATION VIA NUCLEAR VIBRATIONS}

Modulation of the transition frequency leads to a nuclear response not only at the unperturbed resonant nuclear transition frequency, $\omega_{21}$, but also at the frequencies shifted from it by the multiple vibration frequency $\pm n \Omega$ (where $n$ is an integer number $)^{8,9}$. Typically, the intensities of the observed sidebands are small as compared to the central component. However, in the case of the uniform nuclear vibrations, the intensities of the central line and sidebands are defined by the Bessel functions of the first kind, $J_{n}^{2}(m)$, where $m=2 \pi R / \lambda$, and may be comparable to each other ${ }^{10,11}$, as shown in Fig 2 .

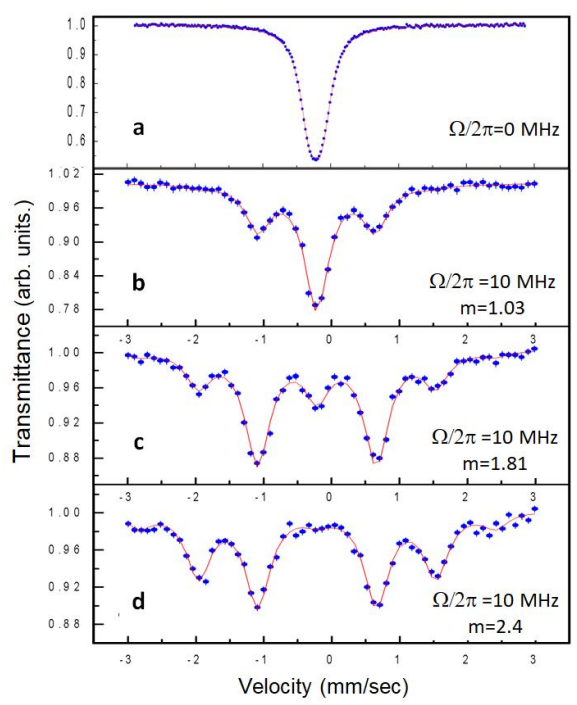

Figure 2. (Color online) Measured Mössbauer transmission spectra (the blue dots with error bars) of a stainless-steel foil of $25 \mu \mathrm{m}$ physical thickness (the effective optical thickness is $t_{A}=5.18$ ), piston-like vibrated with frequency of $10 \mathrm{MHz}$ at various vibration amplitudes corresponding to the modulation indexes $m=2 \pi R / \lambda: a-m=0 ; b-m=1.03 ; c-m=1.81 ; d$ $m=2.40$. The red curves are the theoretical plots in assumption of the piston-like absorber vibration. 
The dependence of the intensities of spectral lines on the modulation index is shown in Fig.3. It is important to note that for a specific value of the modulation index, $m \approx 2.4$, no response is present at the unperturbed transition frequency due to the fact that $J_{n}^{2}(2.4) \approx 0$, as shown in Fig.2d and Fig.3.

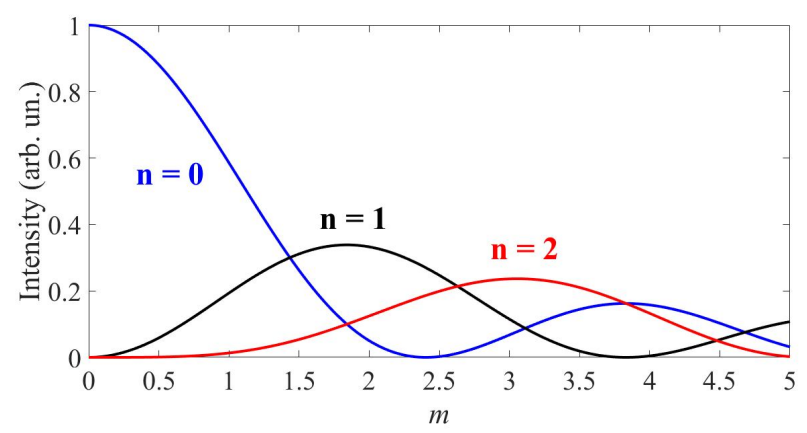

Figure 3. (Color online) Dependence of the intensity of the central line $(n=0)$ of the Mössbauer transmission spectrum and two nearby satellites $(n=1,2)$ on the modulation index $m=2 \pi R / \lambda$.

Such a technique allows to extend a spectrum of the incident radiation by producing the vibrational sidebands as a result of the coherent forward scattering of the incident photon in the vibrated absorber ${ }^{11}$. In its turn, such a spectral extension allows for higher transmission of the total energy of the incident photon through the absorber, since the sidebands don't experience resonant absorption ${ }^{11}$.

In the reference frame accompanying the vibrated nuclei, the nuclear transition is unperturbed, whereas the incident photon is seen as a pure frequency modulated field corresponding to the spectral comb structure without any amplitude modulation. When one of its spectral components is tuned into the resonance with the unperturbed nuclear transition it gets absorbed corresponding to the appearance of the amplitude modulation of an output photon. For certain values of the modulation index, it leads to formation of the regular train of pulses (Fig.4a) from the incident smooth wave packet, which peak amplitude and shape depend on the vibration phase with respect to the front of the single-photon wave packet and the vibration frequency.

In the case when the incident photon is detuned from the unperturbed resonance by the $+\Omega$ or $-\Omega$ (i.e., tuned into the first positive or negative sideband) and the magnitude of that sideband is maximized (which corresponds to the modulation index $m \approx 1.8$ ), a train of the short pulses is formed as it was experimentally demonstrated recently ${ }^{12}$. Physically, it corresponds to the situation when phases of the central component and one of the two first sidebands as well as of both second sidebands of the incident field are synchronized, while the other one of the two first sidebands (possessing a "wrong" phase) is resonantly absorbed. In-phase spectral components separated from each other by the vibration frequency naturally form a train of short pulses with the pulse duration defined by the reciprocal of the product of the modulation frequency and the number of such components. In the case when an incident photon is tuned into the second or third sideband and the magnitude of the corresponding sideband is maximized (which corresponds to the modulation index $m \approx 3.1$ and $m \approx 4.2$, accordingly), each pulse in the sequence is further split into two or three pulses resulting in formation of the regular bunches of such pulses ${ }^{13}$. As was recently suggested, such short bunches of pulses may be used for the realization of the quantum memory with the hard $\mathrm{x}$-ray photons at the nuclear transitions ${ }^{14}$.

If the source frequency coincides with the unperturbed absorber nuclear frequency and the vibration amplitude is equal to $R \approx 0.38 \lambda$ (in the case of $14.4 \mathrm{keV}$ transition of ${ }^{57} \mathrm{Fe}$, one has $R \approx 0.33 \AA$ ), the modulation index is $m \approx 2.4$ resulting in disappearance of the central absorption line (Fig.2a,d). In this case, the photon acquires just a small quasi-harmonic amplitude modulation of the waveform, which value vanishes with increasing the modulation frequency, Fig.4b. Thus, at high enough vibration frequency of the absorber, the waveform of the transmitted photon remains practically unaltered. This is the regime of acoustically induced transparency (AIT) ${ }^{15}$, which was experimentally demonstrated recently ${ }^{16}$. Physically, it corresponds to the situation when the single spectral line of the absorber is split into sidebands owing to 
vibration. At the vibration amplitude corresponding to $m \approx 2.4$, a spectral window of transparency appears in the place of the absorption line with a width equal to twice the modulation frequency (similar to the ATS splitting). In this case, the whole spectrum of the incident photon $\left(\Delta_{s}\right)$ falls into such a transparency window between the moved apart sidebands, $2 \Omega \gg \Delta_{s}$, and is not affected by the sidebands over the entire propagation length: $2 \Omega \gg>\gamma_{a} t_{a}$ (where $\gamma_{a}$ and $t_{a}$ are the halfwidth of the absorber spectral line and the absorber optical thickness, respectivel)y. Both conditions are fulfilled in the case of sufficiently high vibration frequency.
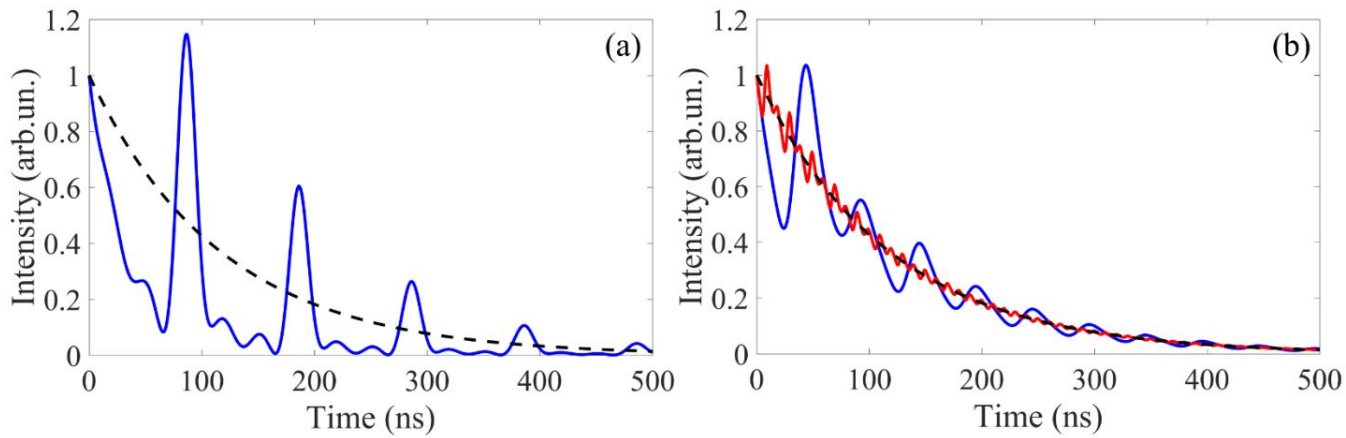

Figure 4. (Color online) Waveform (the time dependence of the photon detection probability proportional to the intensity of the single-photon wave packet) of the $14.4-\mathrm{keV}$ photon transmitted through the vibrating ${ }^{57} \mathrm{Fe}$ stainless-steel absorber, compared to the incident photon (black dashed line) in the case of the photon with the Lorentz spectrum. The blue line in (a) corresponds to the absorber vibration frequency of $10 \mathrm{MHz}$, detuning between the source and absorber spectral lines of $10 \mathrm{MHz}$ (tuning the incident field to the first sideband of the Mössbauer spectrum of the vibrating absorber in Fig.2d), the modulation index of $m=1.84$, and the initial vibration phase (the vibration phase at the front of the waveform) of $\pi / 4$. The blue line in (b) corresponds to the absorber vibration frequency of $10 \mathrm{MHz}$, the resonance between the source and absorber spectral lines, the modulation index of $m=2.4$, and the initial vibration phase of $\pi / 4$. The red line in (b) is plotted for the absorber vibration frequency of $50 \mathrm{MHz}$ with the same values of other parameters.

In both above cases, if the initial phase of vibration (the vibration phase at the moment when the front of the photon waveform is incident to the absorber) is undefined (vibration of the absorber is not synchronized with formation of photons in the source), then averaging over the initial vibration phase takes place. This results in vanishing of a regular amplitude modulation in the photon waveform (Fig.5).
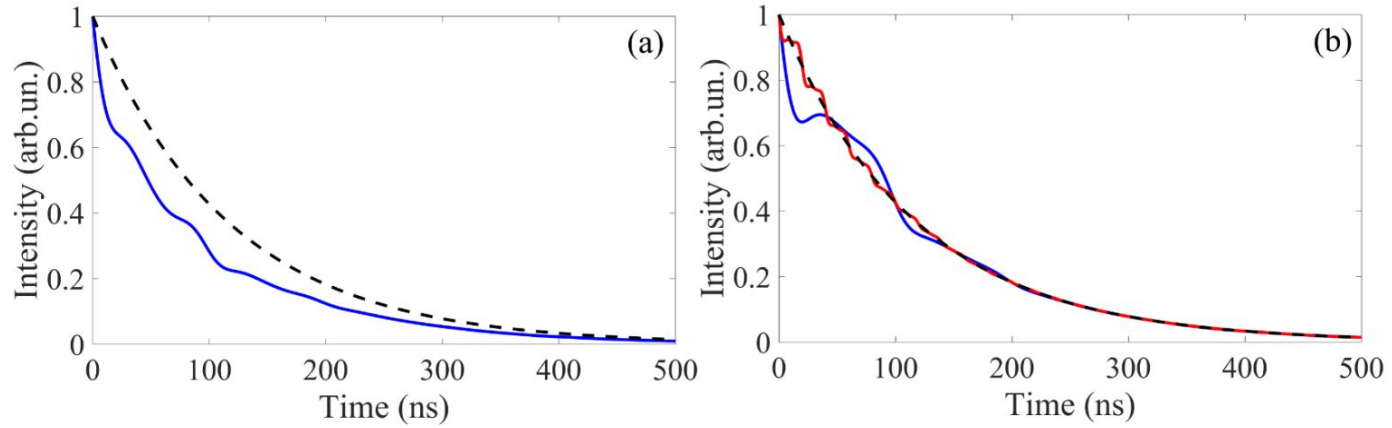

Figure 5. (Color online) Waveform of the $14.4-\mathrm{keV}$ photon transmitted through the vibrating ${ }^{57} \mathrm{Fe}$ stainless-steel absorber compared to the incident photon (black dashed line) in the case of the photon with Lorentz spectrum. The blue line in (a) corresponds to that in Fig.4a, i.e., the absorber vibration frequency is $10 \mathrm{MHz}$, detuning between the source and absorber spectral lines is $10 \mathrm{MHz}$ (tuning the incident field to the first sideband of the Mössbauer spectrum of the vibrating absorber in Fig.2d), the modulation index is $m=1.84$, but the initial vibration phase is averaged over $2 \pi$. The blue and red lines in (b) correspond to those in Fig.4b, i.e., the absorber vibration frequency is $10 \mathrm{MHz}$ and $50 \mathrm{MHz}$, respectively, the source and absorber spectral lines coincide, the modulation index is $m=2.4$, but the initial vibration phase is averaged over $2 \pi$.

However, in the case described by Fig.5a, intensity of the output photon at any moment of time is less than that of the incident photon (blue curve in Fig.5a is below the black one) at any vibration frequency since one of the spectral 
components is absorbed resulting in loss of the total energy of the photon. In the case of AIT (Fig.5b), the energy loss is almost absent. In fact, at some moments of time an intensity of an output single-photon wave packet exceeds, while at other moments it is below intensity of the incident photon due to the remaining dispersion of the absorber at the wings of the spectral sidebands. Similar to the case when the vibration phase is locked to the front of the single-photon wave packet (Fig.4b), a difference between the waveforms of the incident and outcoming photons becomes negligible for sufficiently high vibration frequency.

\section{CONCLUSION}

In this work, we briefly discussed the coherent acoustic modulation of the nuclear transition frequency as an efficient method of controlling the spectral and temporal characteristics of the x-ray single photons propagating through the optically thick resonant medium. We illustrated it with a specific example of controlling spectral-temporal characteristics of the 14.4-keV single-photon wave packet produced by the radioactive or synchrotron Mössbauer source via its resonant interaction with the acoustically vibrated ${ }^{57} \mathrm{Fe}$ nuclear absorber.

It is worth noting that the considered technique of the atomic transition frequency modulation is rather general. In particular, as shown in our recent works ${ }^{17-19}$, it can be used also for generation of the attosecond extreme ultraviolet and $\mathrm{x}$-ray pulses or amplification of the attosecond pulses produced via HHG in the active medium of a plasma-based x-ray laser modulated by a strong infrared laser field.

\section{ACKNOWLEDGEMENTS}

We acknowledge support from the National Science Foundation (NSF, Grant No. PHY-182-09-30), the Russian Foundation for Basic Research (RFBR, Grant No.19-02-00852). The numerical studies presented in this paper were supported by the Russian Science Foundation (RSF, Grant No. 19-72-00140). I.R.Kh. acknowledges support by the Foundation for the Advancement of Theoretical Physics and Mathematics BASIS.

\section{REFERENCES}

[1] Adams, B. W., Buth, C., Cavaletto, S. M., Evers, J., Harman, Z., Keitel, C. H., Palffy, A., Picon, A., Röhlsberger, R., Rostovtsev, Y., Tamasaku, K., "X-ray quantum optics," J. Mod. Opt. 60(1), 2-21 (2013).

[2] Kuznetsova, E., Kocharovskaya, O., "Quantum optics with X-rays," Nat. Photon. 11, 685-686 (2017).

[3] Autler, S. H., Townes, C. H., "Stark Effect in Rapidly Varying Fields," Phys. Rev. 100(2), 703-722 (1955).

[4] Boller, K. J., İmamoğlu, A., Harris, S. E., "Observation of Electromagnetically Induced Transparency," Phys. Rev. Lett. 66(20), 2593(4) (1991).

[5] Vagizov, F., "The splitting of hyperfine lines of ${ }^{57}$ Fe nuclei in RF magnetic field," Hyperfine Interact. 61, 13591362 (1990).

[6] Coussement, R., Rostovtsev, Y., Odeurs, J., Neyens, , G., Muramatsu, H., Gheysen, S., Callens, R., Vyvey, K., Kozyreff, G., Mandel, P., Shakhmuratov, R., Kocharovskaya, O., "Controlling Absorption of Gamma Radiation via Nuclear Level Anticrossing," Phys. Rev. Lett. 89(10), 107601(4) (2002).

[7] Röhlsberger, R., Wille, H.-C., Schlage, K., Sahoo, B., "Electromagnetically induced transparency with resonant nuclei in a cavity," Nature (London) 482, 199-203 (2012).

[8] Ikonen, E., Helistö, P., Katila, T., Riski, K., "Coherent transient effects due to phase modulation of recoilless $\gamma$ radiation," Phys. Rev. A 32(4), 2298-2315 (1985).

[9] Tsankov, L. T., "Experimental observations on the resonant amplitude modulation of Mossbauer gamma rays," J. Phys. A: Math. Gen. 14, 275 (1981). 
[10] Mkrtchyan, A. R., Arutyunyan, G. A., Arakelyan, A. R., Gabrielyan, R. G., "Modulation of Mössbauer radiation by coherent ultrasonic excitation in crystals," Phys. Status Solidi (b) 92(1), 23-29 (1979).

[11] Shvyd'ko, Yu. V., Smirnov, G. V., "Enhanced yield into the radiative channel in Raman nuclear resonant forward scattering," J. Phys.: Condens. Matter 4, 2663 (1992).

[12] Vagizov, F., Antonov, V., Radeonychev, Y. V., Shakhmuratov, R. N., Kocharovskaya, O., "Coherent control of the waveforms of recoilless $\gamma$-ray photons," Nature(London) 508, 80-83 (2014).

[13] Shakhmuratov, R. N., Vagizov, F. G., Antonov, V. A., Radeonychev, Y. V., Scully, M. O., Kocharovskaya, O., "Transformation of a single-photon field into bunches of pulses," Phys. Rev. A 92(2), 023836(15) (2015).

[14]Zhang, X., Liao, W.-T., Kalachev, A. A., Shakhmuratov, R. N., Scully, M. O., Kocharovskaya, O., "Nuclear quantum memory and time sequencing of a single $\gamma$-photon," Phys. Rev. Lett. 123(25), 250504(5) (2019).

[15] Radeonychev, Y. V., Tokman, M. D., Litvak, A. G., Kocharovskaya, O., "Acoustically Induced Transparency in Optically Dense Resonance Medium," Phys. Rev. Lett. 96(9), 093602(4) (2006).

[16] Radeonychev, Y. V., Khairulin, I. R., Vagizov, F. G., Scully, M. O., Kocharovskaya, O., "Observation of Acoustically Induced Transparency for $\gamma$-Ray Photons," Phys. Rev. Lett. 124(16), 163602(7) (2020).

[17] Antonov, V. A., Han, K. Ch., Akhmedzhanov, T. R., Scully, M., Kocharovskaya, O., "Attosecond Pulse Amplification in a Plasma-Based X-Ray Laser Dressed by an Infrared Laser Field," Phys. Rev. Lett. 123(24), 243903(6) (2019).

[18] Khairulin, I. R., Antonov, V. A., Ryabikin, M. Yu., Kocharovskaya, O., "Sub-fs pulse formation in a seeded hydrogenlike plasma-based x-ray laser dressed by an infrared field: Analytical theory and numerical optimization," Phys. Rev. Res. 2(2), 023255(14) (2020).

[19] Antonov, V. A., Khairulin, I. R., Kocharovskaya, O., "Attosecond-pulse formation in the water-window range by an optically dressed hydrogen-like plasma-based $C^{5+}$ x-ray laser," Phys. Rev. A 102(6), 063528(16) (2020). 